\title{
Fragrant Sesquiterpene Ketones as Trace Constituents from Frankincense Volatile Oil of Boswellia sacra
}

\section{Supporting Information}

Johannes Niebler, ${ }^{\dagger}$ Katharina Zhuravlova, ${ }^{\dagger}$ Mirjana Minceva, $\ddagger$ and Andrea Buettner*, ${ }^{\dagger}$

${ }^{\dagger}$ Department of Chemistry and Pharmacy, Food Chemistry, Emil Fischer Center, Friedrich-Alexander-

Universität Erlangen-Nürnberg (FAU), Schuhstr. 19, 91052 Erlangen, Germany, andrea.buettner@fau.de,

Tel.: +49 913185 22739, Fax: +498161 491777

‡ Biothermodynamics, TUM School of Life Sciences Weihenstephan, Technische Universität München,

Gregor-Mendel Str. 4, 85354 Freising, Germany

* corresponding author 


\section{GC-MS Characterization of the Volatile Oils:}

Table S1: Constituents in Boswellia sacra Flueck. volatile oil, from Somalia, obtained by hydrodistillation of gum resin material; Primavera Life $\mathrm{GmbH}$, Oy-Mittelberg, Germany.

\begin{tabular}{|c|c|c|c|c|c|}
\hline Nr. & Compound & RI lit. & Reference & RI exp. & $R P A$ \\
\hline 1 & Toluene & $<800$ & & & $0.09 \%$ \\
\hline 2 & $\begin{array}{l}\text { Hashishene (5,5-Dimethyl-1- } \\
\text { vinylbicyclo[2.1.1]hexane) }\end{array}$ & 924 & & & $0.30 \%$ \\
\hline 3 & Tricyclene & 921 & 1 & 923 & $0.09 \%$ \\
\hline 4 & $\alpha$-Thujene & 924 & 1 & 930 & $1.54 \%$ \\
\hline 5 & $\alpha$-Pinene & 942 & 2 & 937 & $20.23 \%$ \\
\hline 6 & Camphene & 946 & 1 & 951 & $1.45 \%$ \\
\hline 7 & Thuja-2,4(10)-diene & 953 & 1 & 954 & $0.07 \%$ \\
\hline 8 & Sabinene & 969 & 1 & 974 & $4.27 \%$ \\
\hline 9 & $\beta$-Pinene & 974 & 1 & 979 & $1.67 \%$ \\
\hline 10 & $\beta$-Myrcene & 988 & 1 & 990 & $1.95 \%$ \\
\hline 11 & 8-3-Carene & 1008 & 1 & 1009 & $0.48 \%$ \\
\hline 12 & p-Cymene & 1020 & 1 & 1026 & $6.57 \%$ \\
\hline 13 & Limonene & 1024 & 1 & 1031 & $10.10 \%$ \\
\hline 14 & Eucalyptole (1,8-Cineol) & 1026 & 1 & 1034 & $0.35 \%$ \\
\hline 15 & Oxygenated Monoterpene & 1049 & 2 & 1050 & $0.03 \%$ \\
\hline 16 & cis-Sabinene hydrate & 1065 & 1 & 1071 & $0.10 \%$ \\
\hline 17 & Oxygenated Monoterpene & - & & 1087 & $0.20 \%$ \\
\hline 18 & Perillene & 1102 & 1 & 1097 & $0.20 \%$ \\
\hline 19 & $\alpha$-Pinene oxide & 1099 & 1 & 1100 & $1.17 \%$ \\
\hline 20 & trans-Sabinene hydrate & 1098 & 1 & 1101 & $0.07 \%$ \\
\hline 21 & $\begin{array}{l}\text { Isoamyl isovalerat (3- } \\
\text { methylbutyl 3- } \\
\text { methylbutanoat) }\end{array}$ & 1100 & 1 & 1106 & $0.09 \%$ \\
\hline 22 & Oxygenated Monoterpene & 1108 & 2 & 1111 & $1.48 \%$ \\
\hline 23 & trans-Thujone ( $\beta$-Thujone) & 1112 & 1 & 1119 & $0.12 \%$ \\
\hline 24 & trans-p-Mentha-2,8-dienol & 1119 & 1 & 1122 & $0.15 \%$ \\
\hline 25 & $\alpha$-Campholenal & 1122 & 1 & 1128 & $0.86 \%$ \\
\hline 26 & cis-Limomene epoxide & 1132 & 1 & 1133 & $0.49 \%$ \\
\hline 27 & trans-Limonene epoxide & 1137 & 1 & 1137 & $0.48 \%$ \\
\hline 28 & trans-Pinocarveol & 1135 & 1 & 1142 & $1.16 \%$ \\
\hline 29 & trans-Verbenol & 1137 & 1 & 1147 & $2.03 \%$ \\
\hline 30 & trans-Pinocamphone & 1158 & 1 & 1161 & $0.21 \%$ \\
\hline 31 & $\begin{array}{l}\text { Pinocarvone (2(10)-Pinen-3- } \\
\text { one) }\end{array}$ & 1160 & 1 & 1163 & $0.27 \%$ \\
\hline 32 & cis-Sabinol & 1170 & 2 & 1170 & $0.07 \%$ \\
\hline 33 & 4-Terpineol & 1182 & 2 & 1182 & $0.32 \%$ \\
\hline 34 & p-Methylacetophenone & 1186 & 2 & 1186 & $0.04 \%$ \\
\hline 35 & p-Cymen-8-ol & 1187 & 2 & 1188 & $0.64 \%$ \\
\hline 36 & $\alpha$-Terpineol & 1193 & 2 & 1196 & $0.95 \%$ \\
\hline 37 & Myrtenol & 1198 & 2 & 1195 & $0.25 \%$ \\
\hline 38 & Verbenone & 1204 & 1 & 1208 & $1.80 \%$ \\
\hline 39 & trans-Carveol & 1215 & 1 & 1220 & $0.86 \%$ \\
\hline 40 & cis-Carveol & 1230 & 2 & 1234 & $0.10 \%$ \\
\hline 41 & Carvone & 1239 & 1 & 1245 & $0.81 \%$ \\
\hline 42 & 3,5-Dimethoxytoluene & 1264 & 2 & 1265 & $0.35 \%$ \\
\hline 43 & Bornyl acetate & 1285 & 1 & 1285 & $0.96 \%$ \\
\hline 44 & $\alpha$-Cubebene & 1348 & 1 & 1347 & $0.40 \%$ \\
\hline 45 & $\alpha$-Ylangene & 1373 & 1 & 1370 & $0.18 \%$ \\
\hline 46 & $\alpha$-Copaene & 1383 & 2 & 1376 & $1.53 \%$ \\
\hline 47 & $\beta$-Bourbonene & 1387 & 1 & 1385 & $0.40 \%$ \\
\hline 48 & $\beta$-Elemene & 1393 & 2 & 1390 & $2.36 \%$ \\
\hline 49 & $\beta$-Caryophyllene & 1426 & 2 & 1422 & $0.15 \%$ \\
\hline 50 & (Z)- $\alpha$-trans-Bergamotene & 1432 & 1 & 1432 & $0.30 \%$ \\
\hline 51 & $\alpha$-Guiaene & 1437 & 1 & 1438 & $0.07 \%$ \\
\hline 52 & Aromadendrene & 1439 & 1 & 1446 & $0.04 \%$ \\
\hline
\end{tabular}




\begin{tabular}{|c|c|c|c|c|c|}
\hline \\
\hline 53 & $\alpha$-Humulene & 1452 & 1 & 1458 & $0.10 \%$ \\
\hline 54 & allo-Aromadendrene & 1458 & 1 & 1462 & $0.33 \%$ \\
\hline 55 & Y-Muurolene & 1478 & 1 & 1475 & $0.66 \%$ \\
\hline 56 & $\beta$-Eudesmene & 1483 & 2 & 1491 & $1.06 \%$ \\
\hline 57 & 4-epi-Cubebol & 1493 & 1 & 1494 & $1.09 \%$ \\
\hline 58 & $\gamma$-Cadinene & 1513 & 1 & 1514 & $0.93 \%$ \\
\hline 59 & Cubebol & 1514 & 1 & 1518 & $1.09 \%$ \\
\hline 60 & trans-Calamenene & 1521 & 1 & 1522 & $0.30 \%$ \\
\hline 61 & Elemol & 1548 & 1 & 1549 & $0.11 \%$ \\
\hline 62 & Oxygenated sesquiterpene & - & & 1553 & $0.40 \%$ \\
\hline 63 & Spatulenol & 1577 & 1 & 1579 & $0.22 \%$ \\
\hline 64 & Caryophyllene oxide & 1582 & 1 & 1585 & $6.24 \%$ \\
\hline 65 & Viridiflorol & 1592 & 1 & 1596 & $0.58 \%$ \\
\hline 66 & Humulene epoxide II & 1608 & 1 & 1612 & $1.46 \%$ \\
\hline 67 & 1,10-diepi-Cubenol & 1618 & 1 & 1616 & $0.32 \%$ \\
\hline 68 & $\tau$-Cadinol (epi- $\alpha$-Cadinol) & 1638 & 1 & 1644 & $0.84 \%$ \\
\hline 69 & $\delta$-Cadinol ( $\alpha$-Muurolol) & 1644 & 1 & 1648 & $0.11 \%$ \\
\hline 70 & Dimer of phellandrene & 1795 & 2 & 1795 & $0.19 \%$ \\
\hline 71 & Cembrene $\mathrm{A}$ & 1965 & 1 & 1955 & $0.13 \%$ \\
\hline 72 & Unidentified diterpene 5 & 2141 & 2 & 2138 & $0.04 \%$ \\
\hline \multirow[t]{2}{*}{73} & $\begin{array}{l}\text { Incensole + Serratol } \\
\text { (coelution) }\end{array}$ & 2152 & 2 & 2151 & $0.43 \%$ \\
\hline & & & & Sum: & $87.53 \%$ \\
\hline
\end{tabular}

Retention indices (RI) measured on a DB-5 column, exp.: experimental values, lit.: values from literature sources, Ref.: reference number, RPA: relative peak area

\section{References for retention indices (table S1):}

(1) Adams, R. P. Identification of Essential Oil Components by Gas Chromatography / Mass Spectrometry, 4th Edition; Allured Business Media: Carol Stream, IL, 2012.

(2) Hamm, S.; Bleton, J.; Connan, J.; Tchapla, A. Phytochemistry 2005, 66, 12, 1499-1514.

Figure S1: GC-MS total ion chromatogram (DB-5 column) of Boswellia sacra volatile oil used in this study. Peak labels refer to table S1.

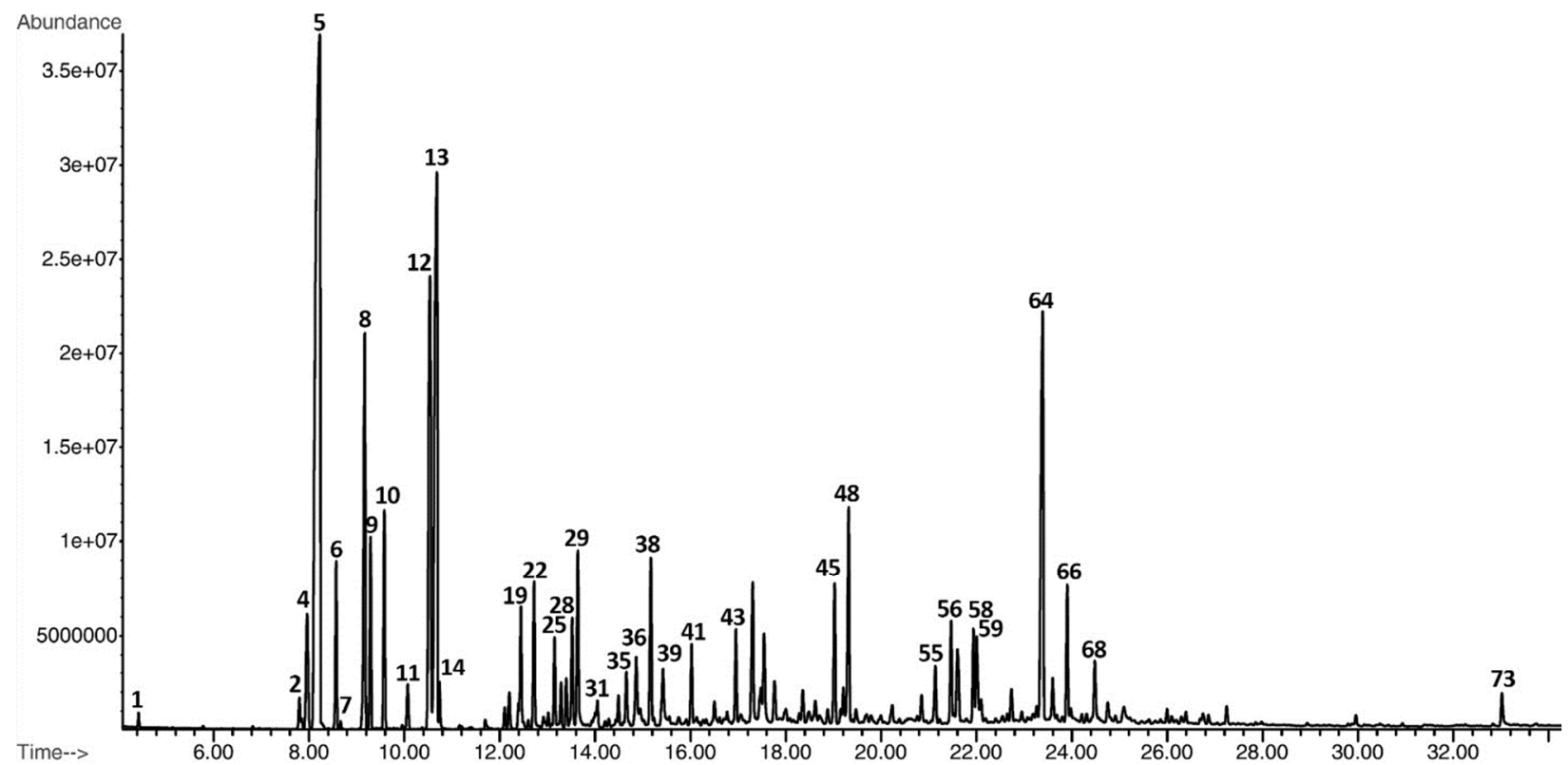


Table S2: Constituents in Cyperus scariosus R.Br. volatile oil, obtained by distillation of rhizomes, Northern India; Essence Pur Ltd., Utting am Ammersee, Germany.

\begin{tabular}{|c|c|c|c|c|c|}
\hline Nr. & Compound & RI lit. & Ref. & RI exp. & RPA \\
\hline 1 & $\alpha$-Pinene & 942 & 1 & 932 & $0.31 \%$ \\
\hline 2 & $\beta$-Pinene & 974 & 1 & 978 & $0.48 \%$ \\
\hline 3 & trans-Pinocarveol & 1135 & 1 & 1143 & $0.25 \%$ \\
\hline 4 & Myrtenal & 1195 & 1 & 1197 & $0.30 \%$ \\
\hline 5 & $\alpha$-Cubebene & 1348 & 1 & 1348 & $0.10 \%$ \\
\hline 6 & Unidentified Compound & & & 1367 & $1.90 \%$ \\
\hline 7 & Cyclosativene & 1371 & 1 & 1372 & $0.08 \%$ \\
\hline 8 & $\alpha$-Copaene & 1374 & 1 & 1379 & $4.37 \%$ \\
\hline 9 & cis- $\beta$-Elemene & 1389 & 1 & 1392 & $0.23 \%$ \\
\hline 10 & Cyperene & 1398 & 1 & 1410 & $14.21 \%$ \\
\hline 11 & $\beta$-Caryophyllene & 1417 & 1 & 1423 & $0.11 \%$ \\
\hline 12 & Norrotundene & & & 1434 & $0.21 \%$ \\
\hline 13 & Cypera-2,4(15)-diene & 1443 & 2 & 1441 & $0.23 \%$ \\
\hline 14 & Spirolepechinene & 1449 & 1 & 1450 & $0.31 \%$ \\
\hline 15 & Rotundene & 1457 & 1 & 1467 & $6.14 \%$ \\
\hline 16 & ү-Gurjunene + Unident. Comp. & 1475 & 1 & 1476 & $2.13 \%$ \\
\hline 17 & Aristolochene & 1487 & 1 & 1487 & $0.16 \%$ \\
\hline 18 & $\beta$-Selinene & 1489 & 1 & 1493 & $2.50 \%$ \\
\hline 19 & Valencene & 1496 & 1 & 1495 & $0.75 \%$ \\
\hline 20 & $\begin{array}{l}\alpha \text {-Selinene }+\alpha \text {-Muurolene }+ \\
\text { Unidentified compound }\end{array}$ & $1498 / 1500$ & 1 & 1500 & $1.23 \%$ \\
\hline 21 & Isorotundene (?) & & & 1509 & $0.94 \%$ \\
\hline 22 & $\delta$-Amorphene & 1511 & 1 & 1520 & $0.93 \%$ \\
\hline 23 & trans-Calamenene & 1521 & 1 & 1524 & $0.94 \%$ \\
\hline 24 & Unidentified Compound & & & 1541 & $1.97 \%$ \\
\hline 25 & $\alpha$-Calacorene & 1544 & 1 & 1544 & $1.01 \%$ \\
\hline 26 & Caryophyllene oxide & 1582 & 1 & 1587 & $3.53 \%$ \\
\hline 27 & $\beta$-Oplopenone & 1607 & 1 & 1607 & $0.97 \%$ \\
\hline 28 & Humulene epoxide II & 1608 & 1 & 1615 & $1.56 \%$ \\
\hline 29 & Unidentified Compound & & & 1622 & $1.94 \%$ \\
\hline 30 & Aristol-9-en-8-one & & & 1628 & $3.88 \%$ \\
\hline 31 & Unidentified Compound & & & 1649 & $2.06 \%$ \\
\hline 32 & Unidentified Compound & & & 1658 & $1.15 \%$ \\
\hline 33 & Unidentified Compound & & & 1663 & $1.04 \%$ \\
\hline 34 & Unidentified Compound & & & 1666 & $1.93 \%$ \\
\hline 35 & Mustakone & 1676 & 1 & 1680 & $4.64 \%$ \\
\hline 36 & Cyperotundone & 1695 & 1 & 1705 & $7.64 \%$ \\
\hline 37 & Unidentified Compound & & & 1709 & $1.71 \%$ \\
\hline 38 & Unidentified Compound & & & 1714 & $1.03 \%$ \\
\hline 39 & $\alpha$-Cyperone & 1752 & 3 & 1750 & $3.09 \%$ \\
\hline 40 & Nootkatone & 1806 & 1 & 1808 & $0.33 \%$ \\
\hline 41 & Cyperadione & 1845 & 2 & 1833 & $0.10 \%$ \\
\hline \multirow[t]{2}{*}{42} & $\begin{array}{l}\text { Di-isooctyl-phthalate (plasticizer } \\
\text { contaminant) }\end{array}$ & 2540 & 4 & 2535 & $5.23 \%$ \\
\hline & & & & Sum: & $83.62 \%$ \\
\hline
\end{tabular}

(?) denominates tentative identifications

Retention indices (RI) measured on a DB-5 column, exp.: experimental values, lit.: values from literature sources, Ref.: reference number, RPA: relative peak area

References for retention indices (table S2):

(1) Adams, R. P. Identification of Essential Oil Components by Gas Chromatography / Mass Spectrometry, 4th Edition; Allured Business Media: Carol Stream, IL, 2012.

(2) Andriamaharavo, N. R. Retention Data. NIST Mass Spectrometry Data Center, 2014.

(3) Morteza-Semnani, K.; Saeedi, M. Flavour Fragr. J. 2005, 20, 3, 332-334.

(4) Kotowska, U.; Zalikowski, M.; Isidorov, V.A. Environ. Monit. Asses. 2012, 184, 5, 2893-2907. 
Figure S2: GC-MS total ion chromatogram of Cyperus scariosus volatile oil used in this study. Numbers refer to compounds from table S2.

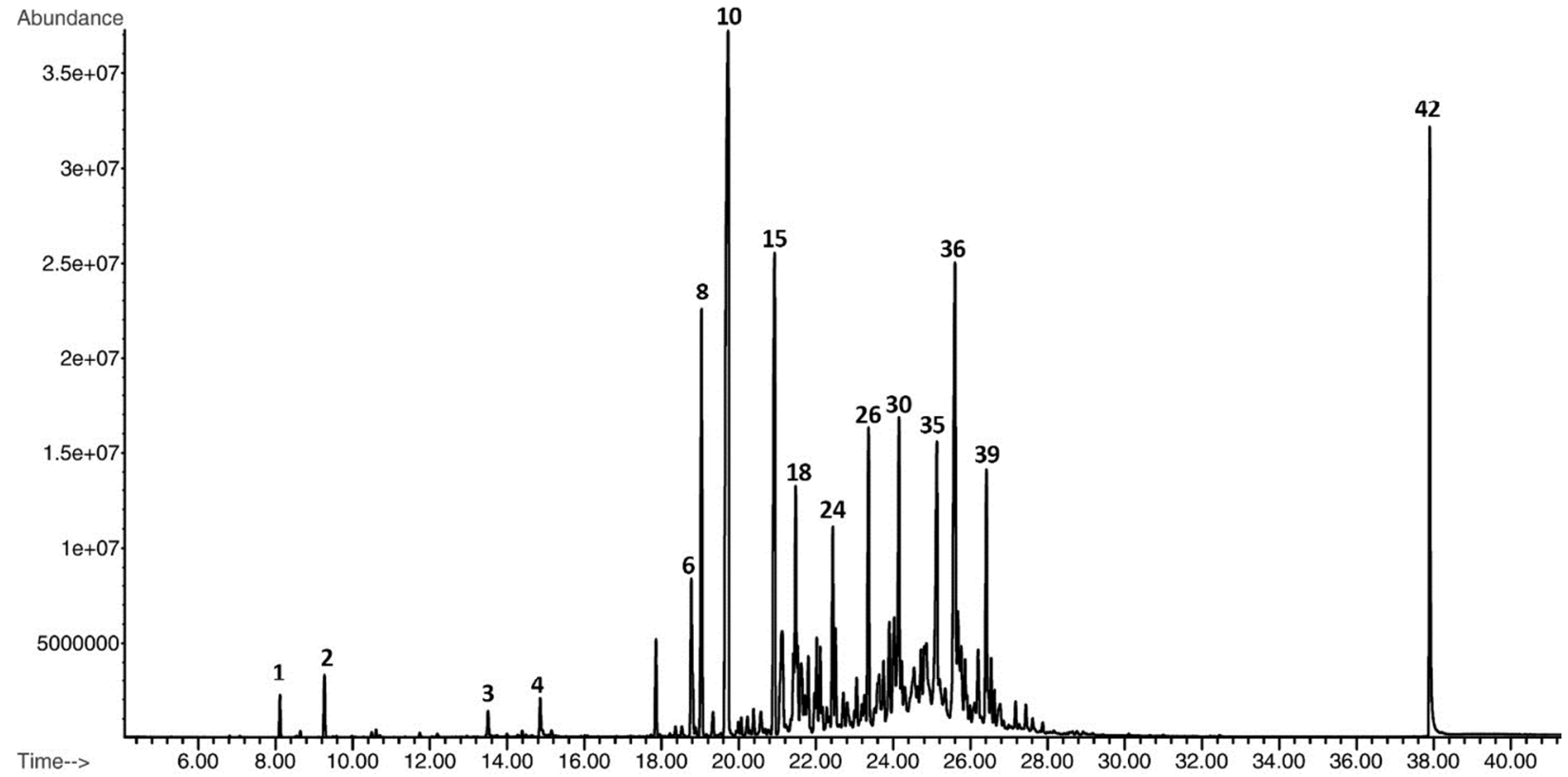




\section{Analytical Data for the Isolated Reference Substance Mustakone (2):}

Mustakone (2): colorless oil, strong woody-spicy odor

${ }^{1} \mathrm{H} \mathrm{NMR}\left(\mathrm{CDCl}_{3}, 600 \mathrm{MHz}\right): \delta 5.75$ (q, J=1.5, $\left.1 \mathrm{H}, \mathrm{H}-3\right), 2.70$ (dd, J=6.6, $\left.1.7 \mathrm{~Hz}, 1 \mathrm{H}, \mathrm{H}-1\right), 2.68(\mathrm{~s}, 1 \mathrm{H}, \mathrm{H}-6)$, 2.02 (d, J=1.5 Hz, $3 \mathrm{H}, \mathrm{H}-14$ ), 1.99 (dd, J=6.8, $1.5 \mathrm{~Hz}, 1 \mathrm{H}, \mathrm{H}-5$ ), 1.87 - 1.92 (m, $1 \mathrm{H}, \mathrm{H}-9$ ), 1.70 - 1.80 (m, 3 $\mathrm{H}, \mathrm{H}-7, \mathrm{H}-8, \mathrm{H}-9$ ), 1.49 - 1.57 (m, $2 \mathrm{H}, \mathrm{H}-8, \mathrm{H}-11$ ), 0.99 (s, $3 \mathrm{H}, \mathrm{H}-15), 0.87$ (d, J=6.8 Hz, $3 \mathrm{H}, \mathrm{H}-12$ ), 0.86 (d, $\mathrm{J}=6.8 \mathrm{~Hz}, 3 \mathrm{H}, \mathrm{H}-13)$;

${ }^{13} \mathrm{C} \mathrm{NMR}\left(\mathrm{CDCl}_{3}, 90 \mathrm{MHz}\right): \delta 204.0$ (C=O, C-2), 169.9 (C, C-4), $121.4(\mathrm{CH}, \mathrm{C}-3), 57.3$ (C, C-10), 56.6 (CH, C1), $56.0(\mathrm{CH}, \mathrm{C}-5), 54.5(\mathrm{CH}, \mathrm{C}-6), 45.4(\mathrm{CH}, \mathrm{C}-4), 36.7\left(\mathrm{CH}_{2}, \mathrm{C}-9\right), 31.8(\mathrm{CH}, \mathrm{C}-11), 23.6\left(\mathrm{CH}_{3}, \mathrm{C}-15\right), 22.0$ $\left(\mathrm{CH}_{2}, \mathrm{C}-8\right), 20.3\left(\mathrm{CH}_{3}, \mathrm{C}-14\right), 19.9\left(\mathrm{CH}_{3}, \mathrm{C}-13\right), 19.5\left(\mathrm{CH}_{3}, \mathrm{C}-12\right)$;

NMR data in agreement with previous reports by Nyasse et al. ${ }^{1}$

EI-MS: $m / z 218$ [M] $^{+}$(13), 175 (86), 161 (37), 148 (52), 147 (100), 135 (38), 133 (57), 122 (67), 119 (38), 105 (66), 91 (53)

HR-MS (ESI-qToF): $m / z[M+H]^{+} 219.1757$ (calculated for $\mathrm{C}_{15} \mathrm{H}_{23} \mathrm{O}^{+} 219.1743$ )

Retention indices ( $n$-alkanes $C_{6}-C_{26}$ ): 1658 (DB-1), 1685 (DB-5), 2260 (DB-WAX), 2255 (DB-FFAP)

$\mathrm{R}_{\mathrm{f}}$ (silica gel, petroleum ether/diethyl ether 80:20): 0.26

\section{Reference:}

(1) Nyasse, B.; Ghogomu, R.; Sondengam, T. B. L.; Martin, M. T.; Bodo, B. Phytochemistry 1988, 27, 10, 3319-3321. 
Figure S3: ${ }^{1} \mathrm{H}$ NMR spectrum for mustakone (2). Impurities are marked, namely peaks for cyperotundone, acetonitrile (HPLC separation) and dichloromethane.

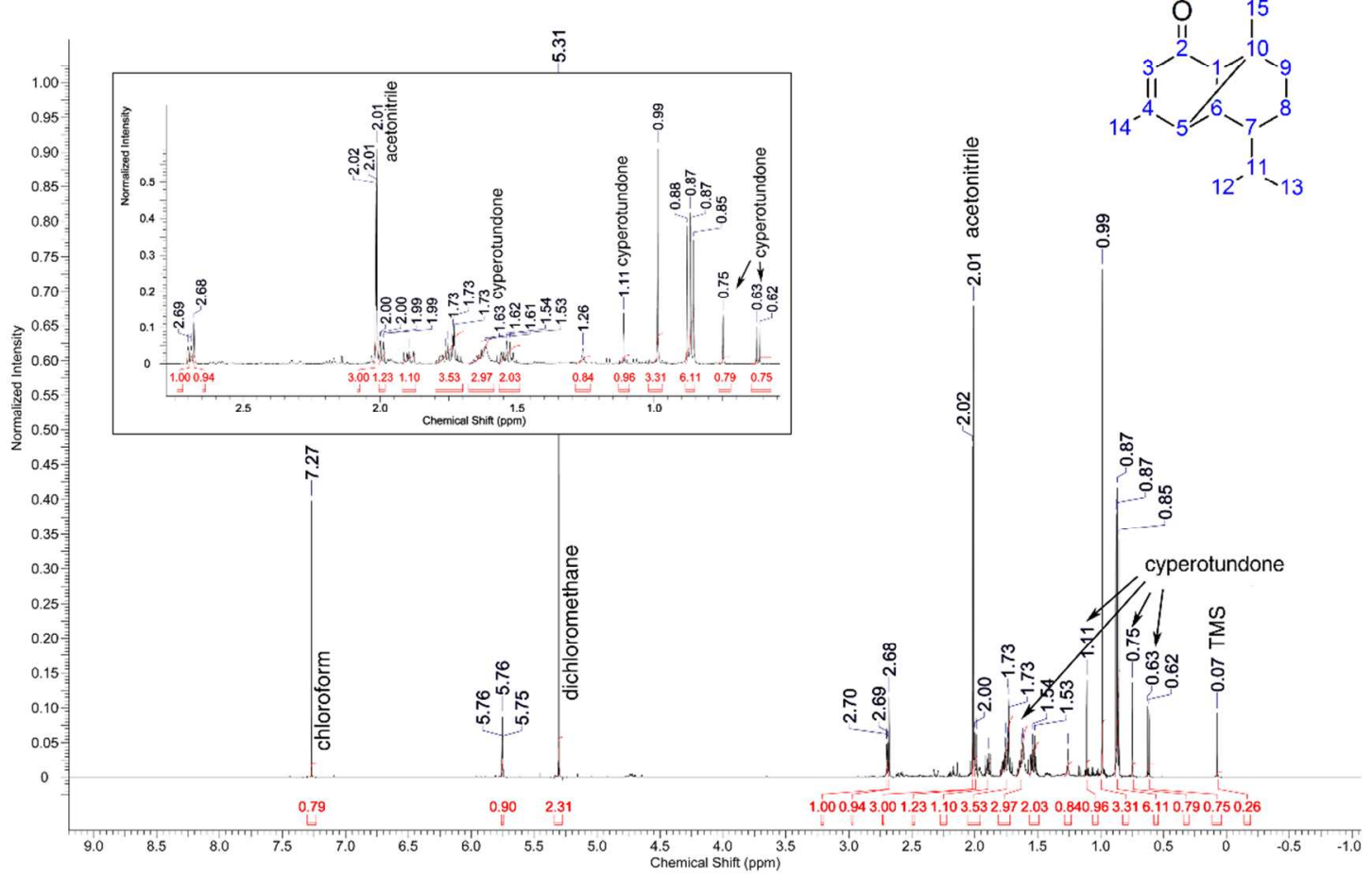

Figure S4: ${ }^{13} \mathrm{C}$ NMR spectrum for mustakone (2). Smaller peaks can mostly be attributed to the main impurity cyperotundone.

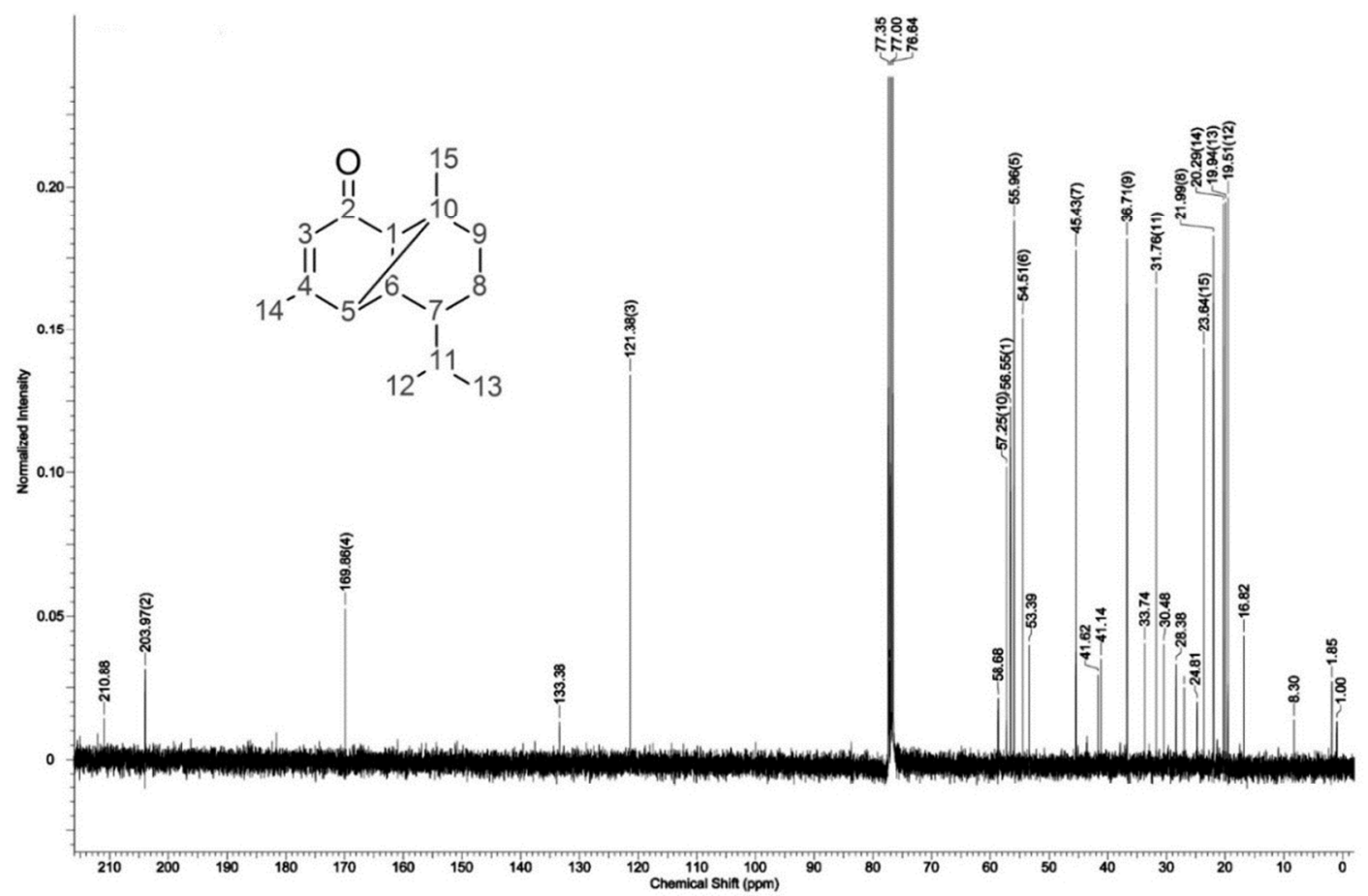


Figure S5: GC-MS total ion chromatogram of the reference substance mustakone (2). The second, closely eluting peak is the impurity of cyperotundone. The two substances could not be separated by preparative HPLC or column chromatography.

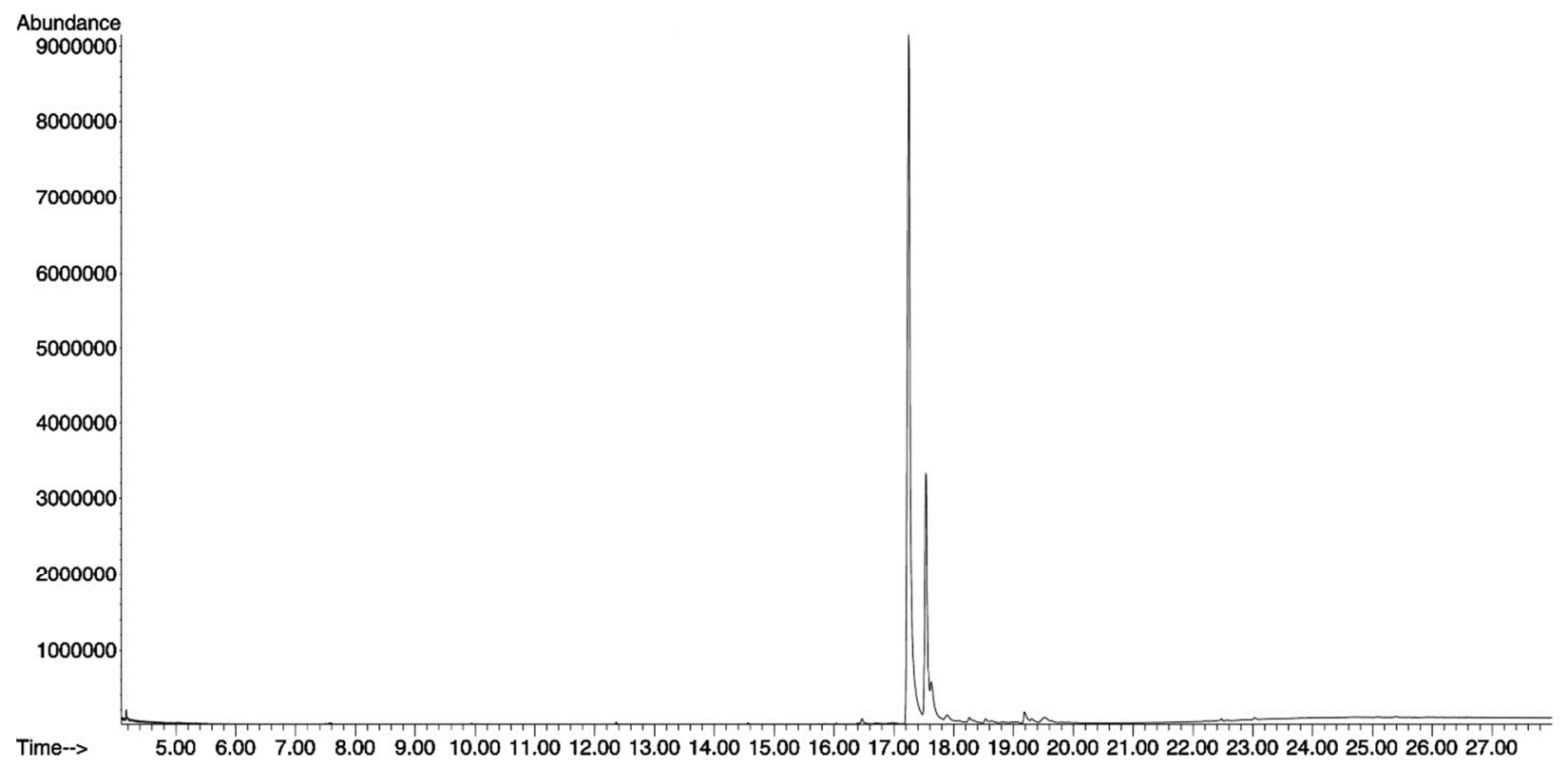

Figure S6: Setup for the preparative gas chromatography

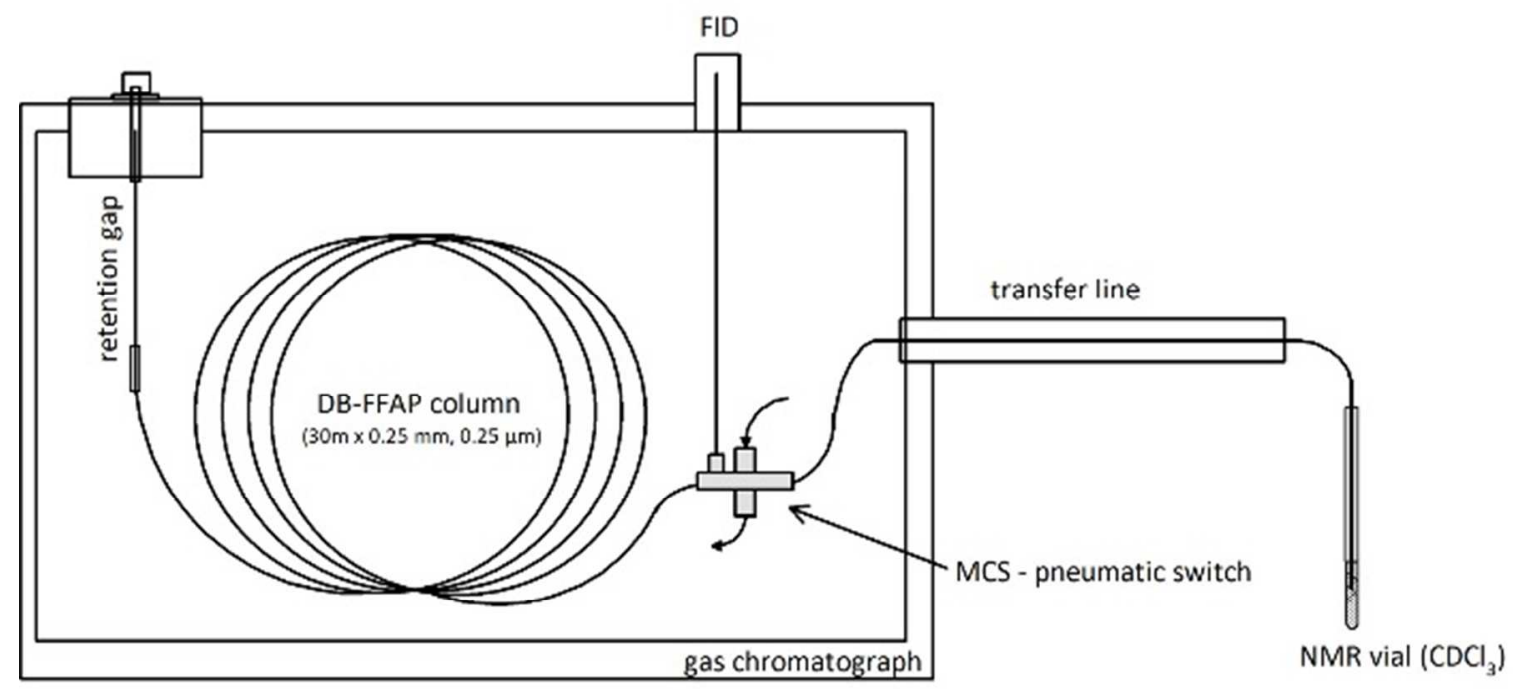

